\title{
COMPETIÇÃO DE MISTURAS DE HERBICIDAS NAS PRINCIPAIS REGIÕES ALGODOEIRAS (Gossypium birsutum L.) NO E. DE MINAS GERAIS
}

\author{
J.P. del C. LACA-BUENDIA*, A.A.C. PURCINO**, \\ L. FERREIRA** \& M.B. FERREIRA*** \\ * Eng. ${ }^{\circ}$ Agr. ${ }^{\circ}$ - Coordenador do Projeto Algodão- \\ EPAMIG. 30.000 - Belo Horizonte, Minas Gerais. \\ ** Eng. ${ }^{\circ}$ Agr. ${ }^{\circ}$, Pesquisador da EPAMIG. 38.100 - \\ Uberaba, Minas Gerais. \\ *** Botânica - Pesquisadora da EPAMIG. 30.000 - \\ Belo Horizonte, Minas Gerais. \\ Trabalho apresentado no III Congresso de la \\ Associación Latinoamericana de Malezas \\ (ALAM) e VIII Reunión de Malezas y su Con- \\ trol (ASAM). Mar del Plata - Argentina, 28 \\ Março - 2 Abril, 1976.
}

Recebido para publicação em 13.06.78.

\section{RESUMO}

Com a finalidade de testar misturas de herbicidas, aplicados em pré-plantio incorporado e préemergência na cultura algodoeira, instala ramse no ano agrícola $74 / 75$, três ensaios. Usaram-se no plantio as cultivares IAC-13-1 (Triângulo e Norte) e Minas Dona Beja (Metalúrgica).

No Triângulo Mineiro, para o total das plantas daninhas somente houve controle até os 30 dias, sendo as melhores misturas: dinitramine + diuron; dinitroanilin + prometryne $\mathrm{e}$ pendimethalin + diuron, sendo esse controle de $96,2 \%, 92,5 \%$ e $96,2 \%$ respectivamente. Com relação aos rendimentos, o melhor tratamento foi pendimetalin + diuron, com $1962 \mathrm{~kg} /$ ha contra 1130 $\mathrm{kg} / \mathrm{ha}$ da testemunha sem capina.

No Norte de Minas Gerais, para o total das plantas daninhas, as melhores misturas foram pendimethalin + diuron com controle de $86,4 \%$ aos 30 dias, $83,6 \%$ aos 50 dias e $70,3 \%$ aos 80 dias após a aplicação. Com relação aos rendimentos as misturas de dinitramine + fluometuron e dinitroanilin + fluometuron produziram respectivamente $1532 \mathrm{~kg} / \mathrm{ha}$ e $1451 \mathrm{~kg} / \mathrm{ha}$ contra $229 \mathrm{~kg} / \mathrm{ha} \mathrm{da}$ testemunha sem capina.

$\mathrm{Na}$ Região Metalúrgica, para o controle to tal das plantas daninhas a mistura mais eficiente foi dinitramine + diuron, com controle de $67 \%$ até os 30 dias após a aplicação. Depois de 50 dias, seu efeito não foi satisfatório. Com relação ao rendi- mento, a mistura dinitramine + fluometuron produziu $831 \mathrm{~kg} / \mathrm{ha}$, contra $145 \mathrm{~kg} / \mathrm{ha}$ da testemunha sem capina.

Em todos os locais a altura das plantas foi afetada pela competição das plantas daninhas. $O$ peso do tapulho e de 100 sementes foram efetados somente na região Metalúrgica, para os tratamentos sem controle sobre as mesmas. Para indice de fibra, percentagem de fibra, comprimento da fibra, indice Pressley, indice Micronaire e maturidade da fibra em nenhuma região foi encontrado efeito negativo da aplicação dos herbicidas.

UNITERMOS: Controle, plantas daninhas, algodão, misturas de herbicidas.

\section{SUMMARY}

Several herbicide mixtures were tested on cotton, (Gossypium hirsutum L.) in the main production areas of the State of Minas Gerais, Brasil. The cultivar "Minas Dona Beta", was used in the Metalúrgica region, whilst in Triângulo and Norte the cultivar employed was ."IAC-13-1", . In Triângu-lo litle regrowth occurred up to 30 days after application when the following mixtures were used: dinitramine + diuron, dinitroanilin + prometryne and pendimethalin + diuron. These treatments controlled $96.2 \%, 92.5 \%$ and $96.5 \%$ of 
the total weeds, respectively. When yields were compared, $1,962 \mathrm{Kg} /$ ha were obtained in the best treatment (pendimethalin + diuron), against the control plots average of $1,130 \mathrm{Kg} / \mathrm{ha}$.

Effective weed control was recorded in North of Minas Gerais when pendimethalin + diuron were applied expressed as: $86.4 \%, 83.6 \%$ and $70.3 \%$ of the total weeds after 30,50 and 80 days, respectively. The mixtures dinitramine + fluometuron and dinitroanilin + fluometuron showed the best results regard to a cotton yield of 1,532 $\mathrm{Kg} / \mathrm{ha}$, produced in the treated plots against only $229 \mathrm{~kg} / \mathrm{ha}$ in the control (unhoed).

The best combination for total weed control in the Metalúrgica region was dinitramine + diuron with an efficiency of $67 \%$ after 30 days. When differences in fiber production were considered, however, the best mixture was dinitramine + fluometuron, the treated plots yielding $831 \mathrm{~kg} / \mathrm{ha}$ and the control $145 \mathrm{~kg} / \mathrm{ha}$.

KEYWORDS: weed control, cotton, mixtu re of herbicides.

\section{INTRODUÇÃO}

A competição das plantas daninhas com as culturas tem grande influência na produtividade, pois elas concorrem por água, luz e nutrientes, além de serem hospedeiras de pragas e doenças. Para o desenvolvimento normal, o algodoeiro exige solos livres das plantas daninhas que também prejudicam o tipo e as características tecnológicas da fibra. A escassez de mão-deobra tem influenciado no uso de herbicidas em todas as culturas industriais, nas quais sua efetivação, devido à sua ação mais rápida e prolongada, resulta mais economica que os procedimentos de capina manual e mecânica.

Estudos realizados indicam que para o controle eficiente de gramineas, tem-se destacado os herbicidas em aplicação de pré-plantio incorporado como trifluralin, dinitramine, nitralin; em aplicação de pré-emergência como alachlor e noruron e em aplicação de pósemergência como DSMA e MSMA, (22, $5,15,3,14,19,9,10,11,25,2,6,7,8$ e 23). Por outro lado, para as plantas daninhas de folhas largas têm-se destacado os herbicidas: diuron, fluometuron, prometryne, norea e linuron $(20,18,21,16$,
$1,25,7$ e 8). Tem-se observado, entretanto, que aplicações destes herbicidas isolados, tanto os específicos para o controle das plantas daninhas de folhas estreitas, como de folhas largas, têm apresentado problemas no controle, is to, porque a eliminação de umas, resulta em um maior desenvolvimento das outras.

Vários estudos têm mostrado que combinações de herbicidas apresentam uma maior eficiência de controle para um maior número de espécies encontradas na cultura algodoeira, devido a que cada um é especialmente eficiente para espécies diferentes de plantas daninhas, tornando-se mais econômico pelo uso de doses menores e pelo prolongamento do poder residual $(1,26,23,24,6,17 \mathrm{e}$ 12).

Pelo visto o melhor controle das plantas daninhas na cultura algodoeira, vai depender do tipo das plantas daninhas predominantes e também das características do solo. Quanto ao tipo do solo em que se encontrava a cultura, foi verificado que os herbicidas foram mais eficientes em solos com calcário (21) assim como, algumas espécies de plantas daninhas apresentaram maior resistência de controle nos solos argilosos, que nos arenosos (18).

O objetivo deste trabalho foi determinar as melhores misturas de herbicidas que sejam mais econômicas e eficientes para o controle do maior número de plantas daninhas e que, por outro lado, não causem efeitos fitotóxicos na cultura e nem afetem o rendimento e a qualidade da fibra do algodoeiro.

\section{MATERIAIS E MÉTODOS}

Estes ensaios foram instalados nas Regiões do Triângulo (Capinópolis, solo argiloso siltoso) Norte (Porteirinha, solo franco arenoso) e na Zona Metalúrgica (Sete Lagoas, solo franco argiloso), cujas análises físicoquímicas encontram-se no quadro 1. 
QUADRO 1 - Análise físico-química dos solos onde foram instalados os experimentos de competição de misturas de herbicidas.

\begin{tabular}{|c|c|c|c|c|c|c|c|c|c|c|c|c|}
\hline Localidade & $\begin{array}{c}\text { Areia } \\
\text { Gross } \\
\% \\
\end{array}$ & $\begin{array}{c}\text { Areia } \\
\text { Fina } \\
\%\end{array}$ & $\begin{array}{c}\text { Silte } \\
\approx \\
\end{array}$ & $\begin{array}{c}\text { Argila } \\
\%\end{array}$ & $\begin{array}{l}\text { Classificaçāo } \\
\text { Textural }\end{array}$ & $\begin{array}{r}\mathrm{pH} \\
\text { Agua } \\
\end{array}$ & $\begin{array}{l}\mathrm{Al}^{3+} \\
\mathrm{eq} \cdot \mathrm{mg} / \\
100 \mathrm{cc} \\
\end{array}$ & $\begin{array}{c}\mathrm{Ca}^{2+} \mathrm{Mg}^{2+} \\
\mathrm{eq} \cdot \mathrm{mg} / \\
100 \mathrm{cc}\end{array}$ & $\begin{array}{c}\mathbf{K} \\
\mathrm{ppm} \\
\end{array}$ & $\begin{array}{c}\mathbf{P} \\
\mathrm{ppm}\end{array}$ & $\begin{array}{l}\text { M.O. } \\
\%\end{array}$ & $\underset{\%}{\mathbf{N}}$ \\
\hline $\begin{array}{l}\text { Capinópolis } \\
\text { Porteirinha } \\
\text { Sete Lagoas }\end{array}$ & $\begin{array}{r}1,0 \\
30,6 \\
20,6\end{array}$ & $\begin{array}{r}3,0 \\
29,6 \\
12,0\end{array}$ & $\begin{array}{l}41,0 \\
23,2 \\
38,0\end{array}$ & $\begin{array}{l}55,0 \\
16,0 \\
29,0\end{array}$ & $\begin{array}{l}\text { Argiloso-siltoso } \\
\text { Franco-arenoso } \\
\text { Franco-argiloso }\end{array}$ & $\begin{array}{l}5,87 \\
6,15 \\
5,15\end{array}$ & $\begin{array}{l}0,00 \\
0,00 \\
0,00\end{array}$ & $\begin{array}{l}9,22 \\
6,11 \\
7,74\end{array}$ & $\begin{array}{l}135^{+} \\
135^{+} \\
117\end{array}$ & $\begin{array}{l}46 \\
22 \\
17\end{array}$ & $\begin{array}{l}2,26 \\
1,59 \\
2,80\end{array}$ & $\begin{array}{l}0,12 \\
0,08 \\
0,14\end{array}$ \\
\hline
\end{tabular}

Análises feitas pelo IPEACO - Seção de Solo, Sete Lagoas - MG . 
Os cultivares utilizados foram o IAC13-1 (Triângulo e Norte) e Minas Dona Beja (Metalúrgica).

O delineamento experimental usado foi blocos casualizados com 10 tratamentos, repetidos cinco vezes. As parcelas eram constituídas de quatro fileiras de 5,0 m de comprimento, espaçadas de $1,0 \mathrm{~m}$ (Triângulo) e 0,8 m (Norte e Metalúrgica), perfazendo uma área de $20 \mathrm{~m}^{2}$ (Triângulo) e $16 \mathrm{~m}^{2}$ (Norte e Metalúrgica), sendo a área útil de $10 \mathrm{~m} 2$ (Triângulo) e $8 \mathrm{~m}^{2}$ (Norte e Metalúrgica).

O plantio foi realizado no dia 06 de novembro de 1974 (Triângulo), 21 de novembro de 1974 (Norte) e 13 de novembro de 1974 (Metalúrgica). As sementes foram colocadas de maneira seguida no sulco, deixando-se cair 30-40 sementes por metro de sulco. A germinação ocorreu no dia 15 de novembro de 1974 (Triângulo), 29 de novembro de 1974 (Norte) e 20 de novembro de 1974 (Metalúrgica). O desbaste foi realizado no dia 02 de dezembro de 1974 (Triângulo) e 12 de dezembro de 1974 (Norte e Metalúrgica).

A adubação foi feita, no momento da semeadura, aplicando-se $50 \mathrm{~kg} / \mathrm{ha}$ de sulfato de amônio, $600 \mathrm{~kg} /$ ha de super- fosfato simples e $40 \mathrm{~kg} / \mathrm{ha}$ de cloreto de potássio (Norte e Metalúrgica) e 60 $\mathrm{kg} / \mathrm{ha}$ no Triângulo Mineiro. Aos 35-40 dias após a ge rminação, foi realizada uma adubação em cobertura com $150 \mathrm{~kg} /$ ha de sulfato de amônio, colocada $0,15 \mathrm{~m}$ ao lado das plantas.

$\mathrm{O}$ ensaio foi mantido sob controle livre do ataque de pragas, fazendo-se 10 a 14 tratamentos fitossanitários, conforme a região onde foram conduzidos os ensaios. Os tratamentos utilizados encontram-se no quadro 2 .

A aplicação dos herbicidas foi realizada com pulverizad or costal, a pressão de $40 \mathrm{lb} / \mathrm{pol}^{2}$, bico Teejet 80.02, malha 100 , a $0,50 \mathrm{~m}$ de altura do solo, com um gasto de 500 litros/ha. Logo após a aplicação dos herbicidas de préplantio, estes foram incorporados ao solo a uma profundidade de 5 a 7 $\mathrm{cm}$, através de duas gradagens cruzadas.

Para o levantamento das plantas daninhas que ocorreram em cada tratamento, foram feitas três contagens de espécies numa área de $0,25 \mathrm{~m}^{2}$, em quatro locais diferentes da área útil de cada parcela, perfazendo uma área total por tratamento estudado de $1 \mathrm{~m}^{2}$. Esta

QUADRO 2 - Tratamentos utilizados com as respectivas doses em $\mathrm{kg}$ do p.c. e do i.a./ha.

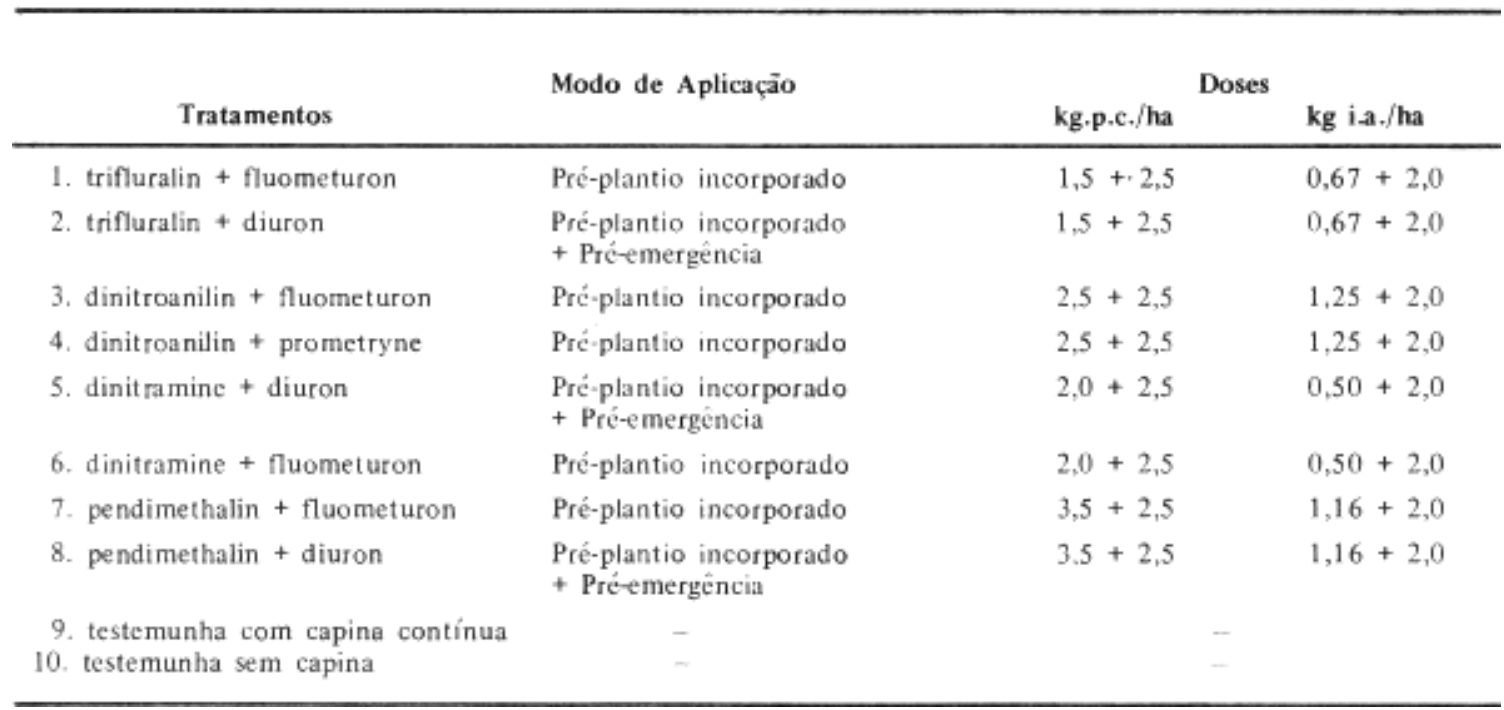


representava $12,5 \%$ da área útil de cada tratamento.

A primeira contagem foi feita aos 32 dias após aplicação dos tratamentos de pré-plantio e 33 dias após aplica ção de pré-emergência; a segunda aos 53 dias após a aplicação de pré-plantio e 54 dias após a aplicação de pré-emergência, e a terceira aos 78 dias após a aplicação dos herbicidas de pré-plantio e 79 dias após a aplicação de pré-emergência.

Foi avaliado o efeito deleté rico dos herbicidas sobre a população das plantas daninhas, utilizando-se a escala de valores adotada pela European Weed Research Council, como descrita por Azzi \& Fernandes (4). 0 indice 1 corres ponde à cobertura total do terreno com plantas daninhas e o indice 9 corresponde à ausência total de plantas daninhas. O indice 6 corresponde a um controle suficiente na prática. Esta avaliação foi feita em três épocas, sendo a primeira, aos 34 dias após a aplicação de pré-plantio e 35 dias após aplicação de pré-emergência; a segunda, aos 54 dias após aplicação de pré-plantio e 55 dias após a pré-emergência e uma terceira, aos 86 dias após a aplicação de préplantio e 87 dias após a pré-emergência.

Foram feitas duas colheitas para a Região do Triângulo, sendo a primeira em 18.04.75 e a segunda em 13.05.75. $\mathrm{Na}$ Região do Norte somente foi realizada uma colheita em 19.04.75 e na Zona Metalúrgica foram feitas duas colheitas, sendo a primeira em 24.04.75 e uma segunda em 16.06.75.
As precipitações pluviométricas durante o ciclo da cultura observadas encontram-se no quadro 3.

\section{RESULTADOS E DISCUSSÃO}

1. Rendimento - Os resultados da análise de variância (quadro 4), mostraram que todos os tratamentos, menos pendimethalin + fluometuron, foram semelhantes à testemunha com capina, para a região do Triângulo Mineiro. Na Região do Norte, as misturas de dinitroanilin + fluometuron, e dinitramine + diu ron foram as únicas que não diferiram significativamente da testemunha com capina. E, na Região Metalúrgica, dinitramine + fluometuron foi melhor que a testemunha sem capina e os outros tratamentos com mistura, um pouco melhor.

2. Altura da Planta - Através da análise de variância, (quadro 4) verifi cou-se que, para a Região do Triângulo, não foi notada nenhuma diferença no porte da planta, em nenhum dos tratamentos estudados. Para a Região do Norte, verificou-se que o porte das plantas foi maior com as aplicações de trifluralin + fluometuron, dinitramine + fluometuron, dinitroanilin + fluometuron e pendimethalin + diuron. E na Região Metalúrgica, verificou-se que trifiuralin + diuron e dinitroanilin + prometryne, foram as únicas misturas diferentes significativamente da testemunha com capina.

QUADRO 3 - Precipitações pluviométricas observadas durante o ciclo da cultura.

\begin{tabular}{|c|c|c|c|c|c|c|c|}
\hline \multirow{2}{*}{ Localidade } & \multicolumn{6}{|c|}{ Meses $(\mathrm{mm})$} & \multirow{2}{*}{ Total } \\
\hline & nov. & dez. & jan. & fev. & mar. & abr. & \\
\hline Capinópolis & 29,0 & 400,0 & 167,8 & 84,2 & 133,0 & 30,0 & 844,4 \\
\hline Porteirinha & 194,8 & 163,8 & 105,8 & 84,4 & 36,8 & 164,0 & 749,6 \\
\hline Sete Lagoas & 54,0 & 352,0 & 182,4 & 167,5 & 43,4 & 88,7 & 888,2 \\
\hline
\end{tabular}


QUADRO 4 - Resultados médios da produção e seus componentes obtidos no ensaio de competição de misturas de herbicidas na cultura algodoeira (G. hirsutum $L$.) no ano agricola de $1974 / 75$.

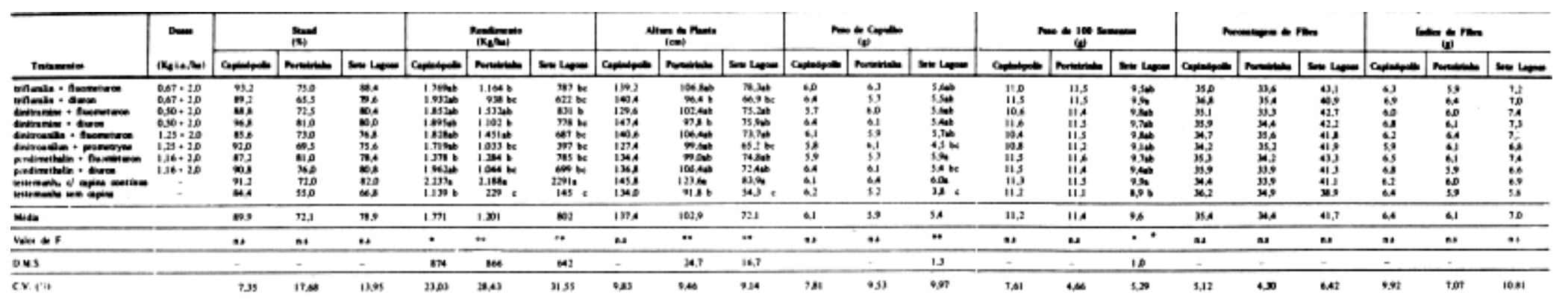

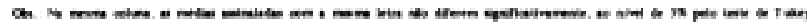

QUADRO 5 - Resultados médios do número total de plantas daninhas por espécie, encontradas numa área de 5,00 m², na cultura algodoeira (G. hirsutum L.) Cultivar IAC-13-1, em Capinópolis, Triângulo Mineiro, no ano agricola de 1974/75.

\begin{tabular}{|c|c|c|c|c|c|c|c|c|c|c|c|c|c|c|c|c|}
\hline \multirow{2}{*}{ Tntamentat } & \multirow{2}{*}{ 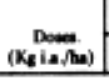 } & \multicolumn{3}{|c|}{ 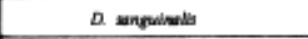 } & \multicolumn{3}{|c|}{$c$ nudiflent } & \multicolumn{3}{|c|}{ Ipomoce top } & \multicolumn{3}{|c|}{ Outra Eaptide is } & \multicolumn{3}{|c|}{ Toted \& Euptiden } \\
\hline & & 1. & 2. & 3. & is & $2 s$ & 3. & 1. & 2. & 3. & 1. & 2. & 3. & 1. & 2.4 & 3. \\
\hline 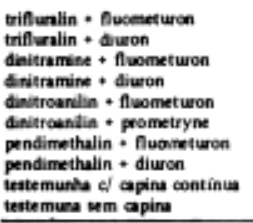 & 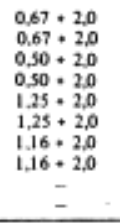 & $\begin{array}{l}1,000 \\
1,000 \\
1,000 \\
1,000 \\
1000 \\
1,000 \\
1000 \\
100 \\
1,000 \\
1,56 \mathrm{~b}\end{array}$ & 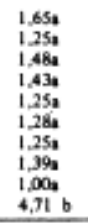 & 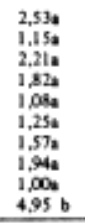 & $\begin{array}{l}1.99 \mathrm{~b} \\
1.46 \mathrm{~b} \\
1.23 \mathrm{~b} \\
1.08 \mathrm{~b} \\
1.59 \mathrm{~b} \\
1.16 \mathrm{~b} \\
1.57 \mathrm{~b} \\
1.08 \mathrm{~b} \\
1.00 \mathrm{~b} \\
1.77 \mathrm{~b}\end{array}$ & 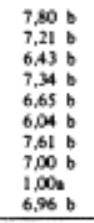 & $\begin{array}{l}10.03 \mathrm{~b} \\
8,15 \mathrm{~b} \\
9.35 \mathrm{~b} \\
9.18 \mathrm{~b} \\
8.06 \mathrm{~b} \\
9.46 \mathrm{~b} \\
9,74 \mathrm{~b} \\
9.68 \mathrm{~b} \\
1.00 \mathrm{~b} \\
10,90 \mathrm{~b}\end{array}$ & $\begin{array}{l}1,160 \\
1,000 \\
1,000 \\
1,000 \\
1,000 \\
1,000 \\
1,000 \\
1,000 \\
1,00 \\
1,44\end{array}$ & $\begin{array}{l}1.36 \\
1,30 \\
1,00 \\
1.00 \\
1,00 \\
1.00 \\
1.00 \\
1,20 \\
1.002 \\
1.63\end{array}$ & $\begin{array}{l}1,39 \\
1,30 \\
1,00 \\
1,08 \\
1,08 \\
1,00 \\
1,00 \\
1,20 \\
1,00 \\
1,83 \\
\end{array}$ & $\begin{array}{l}1,060 \\
1000 \\
1,000 \\
1,000 \\
1080 \\
1000 \\
1,000 \\
1000 \\
1000 \\
1,000 \\
1.736\end{array}$ & 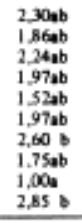 & 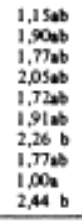 & $\begin{array}{l}1.630 \mathrm{~b} \\
1.464 \mathrm{~b} \\
1.23 \mathrm{a} \\
1.08 \mathrm{a} \\
1.59 \mathrm{ab} \\
1.23 \mathrm{a} \\
1.57 \mathrm{ab} \\
1.06 \mathrm{~b} \\
1.00 \mathrm{a} \\
2,81 \mathrm{~b}\end{array}$ & $\begin{array}{l}8,26 \mathrm{bc} \\
7,51 \mathrm{bc} \\
6,89 \mathrm{~b} \\
7,62 \mathrm{bc} \\
6,82 \mathrm{~b} \\
6,35 \mathrm{~b} \\
8,04 \mathrm{bc} \\
7,27 \mathrm{bc} \\
1,005 \\
9,05 \mathrm{bc}\end{array}$ & $\begin{array}{l}10.47 \mathrm{bc} \\
8.1 \mathrm{~b} \\
8,77 \mathrm{~b} \\
9,52 \mathrm{~b} \\
8,21 \mathrm{~b} \\
9,00 \mathrm{bc} \\
10,08 \mathrm{bc} \\
10,13 \mathrm{bc} \\
1,90 \mathrm{c} \\
9,05 \mathrm{bc} \\
\end{array}$ \\
\hline Nets & & 1,06 & 1.67 & 1.95 & 1.34 & 6,40 & 8,55 & 106 & 1.15 & 1.19 & 1.09 & 2,01 & 1.83 & 1,47 & 0,88 & 8.86 \\
\hline Valor de $F$ & & 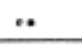 & .. & .. & $\ddot{*}$ & $\cdots$ & 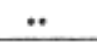 & $\cdot$ & ns & $n *$ & .. & $*$ & $\cdot$ & $\cdots$ & $\infty$ & $*$ \\
\hline DMS. Tukn $5 \mathrm{~s}$ & & $\begin{array}{l}0,29 \\
0,34 \\
\end{array}$ & $\begin{array}{l}1,32 \\
1,56 \\
\end{array}$ & $\begin{array}{l}1,60 \\
1,90 \\
\end{array}$ & $\begin{array}{l}0,69 \\
0,82 \\
\end{array}$ & $\begin{array}{r}2.23 \\
2.65 \\
\end{array}$ & $\begin{array}{r}3,91 \\
4,64 \\
\end{array}$ & 0,46 & - & - & $\overline{0.45}$ & $\begin{array}{l}1,27 \\
1.51 \\
\end{array}$ & $\stackrel{1.24}{-}$ & $\begin{array}{l}0.93 \\
1,11 \\
\end{array}$ & $\begin{array}{l}2,35 \\
2,79 \\
\end{array}$ & $\begin{array}{r}3,52 \\
4,18 \\
\end{array}$ \\
\hline c.V. (x) & & 12,19 & 36,99 & 88.46 & 24,24 & 16.34 & 21,42 & $\infty, 21$ & 34,71 & 40.00 & 16.33 & 29.61 & 31,72 & $D, B$ & 15.99 & 18,58 \\
\hline
\end{tabular}

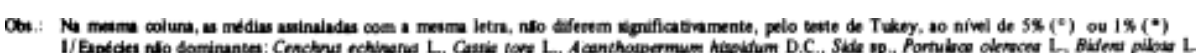

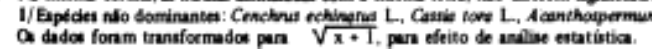


3. Peso de Capulho - Verificou-se que somente para a Região Metalúrgica foram encontradas diferenças significativas, onde se constatou que o peso de tapulho foi menor quando se aplicaram as misturas de dinitroanilin + prometryne e pendimethalin + diuron, que não foram diferentes significativamente da testemunha sem capina, porém um pouco melhor. As demais misturas estudadas não foram diferentes significativamente da testemunha com capina, porém um pouco inferior (quadro 4).

4. Peso de 100 Sementes - Verificou-se que, somente para a Região Metalúrgica, foram encontradas diferenças significativas, tendo sido observado que a mistura de trifluralin + diuron foi a única que apresentou diferença, com a testemunha sem capina (quadro 4).

5. Percentagem de Fibra e Índice de Fibra - Verificou-se que não houve nenhuma diferença significativa para os dados obtidos nas três Regiões estudadas (quadro 4).

6. Número de Plantas Daninhas As análises da variância da transformacão do número de plantas daninhas podem ser vistos nos quadros $5,6 e$ 7. Para a região do Triângulo, verificamos que as espécies dominantes foram: Digitaria sanguinalis (L.) Scop., Commelina nudiflora L. e Ipomoea sp. Nenhuma das misturas estudadas teve um bom controle sobre Commelina nudiflora e Ipomoea sp..

$\mathrm{Na}$ Região Norte, para Sida sp., somente dinitramine + diuron foi inferior, todas as demais misturas foram iguais, estatisticamente, até os 50 dias. Para o controle de Portulaca oleracea, somente as misturas com dinitroanilin e a com pendimethalin + fluometuron, foram inferiores, aos 80 dias. Para Ipomoea sp., todas as misturas com fluometuron for am eficientes. Para as espécies não dominantes a melhor mistura foi a de pendimethalin + diuron.

$\mathrm{Na}$ região Metalúrgica, para o controle de Eleusine indica, até 50 dias, houve controle satisfatório com trifluralin + diuron, dinitramine, diuron, dinitroanilin + prometryne, pendimethalin + fluometuron e pendimethalin + diuron. Todos os tratamentos foram eficientes no controle de Aristida sp, aos 80 dias. Para Ageratum conyzoides, até 50 dias, os melhores resultados foram dados por dinitramine + diuron e pendimethalin + diuron, seguidos de trifluralin + fluometuron, trifluralin + diu ron, dinitroanilin + prometryne e pendimethalin + -fluometuron. Para Sida rhombifolia, até 80 dias, somente dinitroanilin + prometryne foi inferior. Para Sonchus oleraceus, não houve diferenças significativas entre as misturas estudadas. Para Ipomoea sp, as misturas de dinitramine + fluometuron e pendimethalin + fluometuron foram os melhores. Para Portulaca oleracea, o melhor controle foi oferecido por pendimethalin + diuron, aos 80 dias. Para as espécies não dominantes sobressairam trifluralin + diuron e pendimethalin + diuron. Para o total de espécies, aos 30 dias, as piores foram trifluralin + diuron e dinitroanilin + prometryne; os ou tros foram iguais. Aos 80 dias todas as misturas foram iguais.

8. Controle das plantas daninhas pelo método visual da Escala E.W.R.C. (1-9) - Os dados obtidos podem ser observados no quadro 10. Pela análise da variância verifica-se que, para a Região do Triângulo, as melhores misturas foram trifluralin + diuron, dinitroanilin + fluometuron, dinitroanilin + prometry ne e pendimethalin + diuron, não apresentando diferenças significativas da testemunha com capina contínua, até os 85 dias após aplicação.

$\mathrm{Na}$ Região do Norte, dinitramine + fluometuron foi um pouco inferior à testemunha com capina contínua.

$\mathrm{Na}$ Região Metalúrgica, as misturas citadas, até 55 dias, foram pouco inferior à testemunha, e aos 85 dias, todas diferiram significativamente da testemunha. 
QUAD RO 6 - Resultados do total de plantas daninhas por espécies encontradas numa área de 5,00 m $^{2}$, na cultura algodoeira (G. hirsutum L.), cultivar IAC-13-1, em Porteirinha, Norte de Minas, no ano agrícola 1974/75.

\begin{tabular}{|c|c|c|c|c|c|c|c|c|c|c|c|c|c|c|c|c|}
\hline \multirow{2}{*}{ Trutumentiot } & \multirow{2}{*}{ 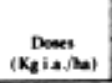 } & \multicolumn{3}{|c|}{ Ses ap. } & \multicolumn{3}{|c|}{ Fortubes ofiences } & \multicolumn{3}{|c|}{ Ipomoed sp. } & \multicolumn{3}{|c|}{ Outras Enptcies II } & \multicolumn{3}{|c|}{ Total at Esptices } \\
\hline & & is & 2. & 3. & 1. & 2. & 3. & 1. & 2. & 3. & 1. & 2. & 3. & 1. & $2 x$ & 3. \\
\hline 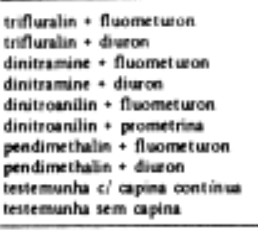 & 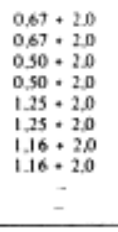 & 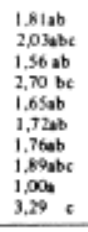 & 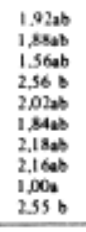 & 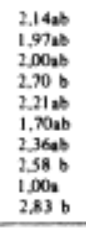 & 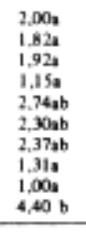 & 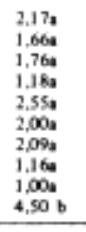 & 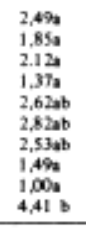 & $\begin{array}{l}1,08 \\
1,00 \\
1,17 \\
1,08 \\
1,08 \\
1,28 \\
1,31 \\
1,37 \\
1,00 \\
1,54 \\
\end{array}$ & $\begin{array}{l}1,08 \\
1,08 \\
1,00 \\
1,25 \\
1,15 \\
1,1,17 \\
1,17 \\
1,08 \\
1,00 \\
1,4\end{array}$ & 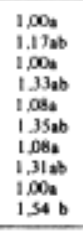 & 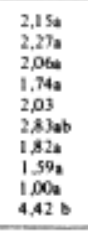 & 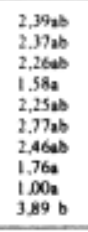 & $\begin{array}{l}2.479 \mathrm{~b} \\
2.542 \mathrm{~b} \\
3.38 \mathrm{~b} \\
2.260 \mathrm{~b} \\
2.360 \mathrm{~b} \\
3.25 \mathrm{~b} \\
2.81 \mathrm{~b} \\
1.99 \mathrm{~b} \\
1.00 \mathrm{a} \\
3.38 \mathrm{~b}\end{array}$ & 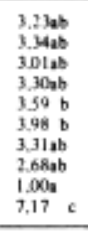 & 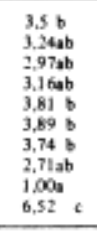 & 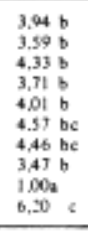 \\
\hline Media & & 1,94 & 1.97 & 2,15 & 2,10 & 2.01 & 2,32 & 1.19 & 1,17 & 1,19 & $2.19 \mathrm{~b}$ & 2.27 & 2,84 & 3.46 & 3,46 & 3.93 \\
\hline Valoe de $\mathrm{F}$ & & 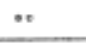 & 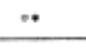 & $\cdot *$ & $*$ & $\cdots$ & $\because$ & $n=$ & $n s$ & 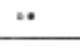 & ¿ & $\because$ & $*$ & $*$ & $*$ & $*$ \\
\hline D.M.S. Tukety $5 \%$ & & $\begin{array}{l}1,25 \\
1,49 \\
\end{array}$ & $\begin{array}{l}1,16 \\
1,38 \\
\end{array}$ & $\begin{array}{l}1.23 \\
1.46 \\
\end{array}$ & $\begin{array}{l}1,96 \\
2,33 \\
\end{array}$ & $\begin{array}{l}1.63 \\
1.94 \\
\end{array}$ & $\begin{array}{l}1.54 \\
1.95 \\
\end{array}$ & - & $\overline{-}$ & $\begin{array}{l}0.48 \\
0.57 \\
\end{array}$ & $\begin{array}{l}1,63 \\
1.93 \\
\end{array}$ & $\begin{array}{l}1,62 \\
1.92 \\
\end{array}$ & $\begin{array}{l}1,36 \\
1,62\end{array}$ & $\begin{array}{l}2,24 \\
2,66 \\
\end{array}$ & $\begin{array}{l}1.89 \\
2.24 \\
\end{array}$ & $\begin{array}{l}1.49 \\
1.77\end{array}$ \\
\hline CV. (\$) & & 30,21 & 27,62 & 26.76 & 43.65 & 38.13 & 33,82 & 24.54 & 21,02 & 19,11 & 40,77 & 33.10 & 25,07 & 30.30 & 25.49 & 17.79 \\
\hline
\end{tabular}

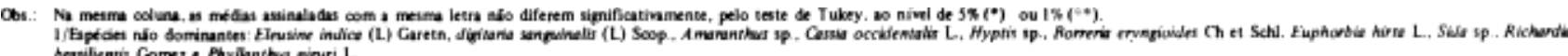

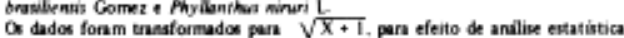

QUADRO 7 - Resultados médios do número total de plantas daninhas por espécies encontradas numa área de $5,00 \mathrm{~m}^{2}$, na cultura algodoeira $(G$. hirsutum L.), cultivar «Minas Dona Beja. em Sete Lagoas, Zona Metalúrgica, Minas Gerais, no ano agricola de $1974 / 75$.

\begin{tabular}{|c|c|c|c|c|c|c|c|c|c|c|c|c|c|c|c|c|}
\hline \multirow{2}{*}{ Tntamento: } & \multirow{2}{*}{ 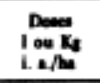 } & \multicolumn{3}{|c|}{ Eleusine inditio } & \multicolumn{3}{|c|}{ Aritrib op } & \multicolumn{3}{|c|}{ Aorroum congsobles } & \multicolumn{2}{|c|}{ Sale ap } & \multirow[b]{2}{*}{ 3. } & \multicolumn{3}{|c|}{ Sanchur olenucee } \\
\hline & & 1. & 2. & 3. & 1. & 2. & 3. & I. & 2. & 3. & 1. & 2. & & I. & 2. & 3. \\
\hline 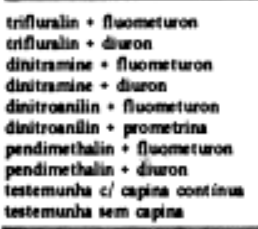 & 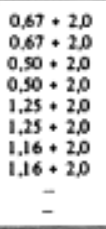 & $\begin{array}{l}1,2506 \\
1,060 \\
1000 \\
1000 \\
1006 \\
1000 \\
1000 \\
1000 \\
1000 \\
1,81 \mathrm{~b}\end{array}$ & 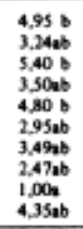 & $\begin{array}{l}6,76 \mathrm{~b} \\
5,38 \mathrm{~b} \\
7,400 \mathrm{~b} \\
5,33 \mathrm{~b} \\
6,22 \mathrm{~b} \\
4.19 \mathrm{~b} \\
4.73 \mathrm{~b} \\
3,8.12 \mathrm{~b} \\
1.00 \mathrm{~b} \\
6,09 \mathrm{~b}\end{array}$ & 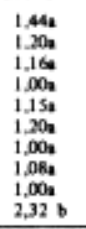 & 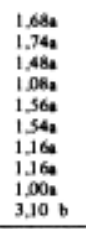 & 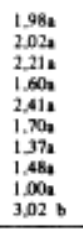 & $\begin{array}{l}1, \infty \\
1,00 \\
1,0 \\
1,00 \\
1,0 \\
1,72 \\
1,00 \\
1,00 \\
1,00 \\
1,20\end{array}$ & 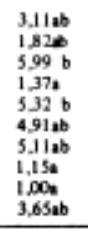 & 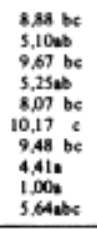 & 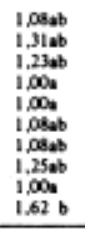 & 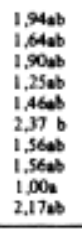 & 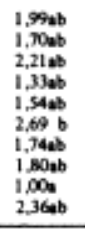 & $\begin{array}{l}1,00 \\
1,43 \\
1,20 \\
1,36 \\
1,16 \\
1,31 \\
1,15 \\
1,25 \\
1,0 \\
1,31 \\
\end{array}$ & $\begin{array}{l}1,78 \\
2.16 \\
1,99 \\
1,95 \\
1,65 \\
1,93 \\
2.23 \\
1,73 \\
1,00 \\
1,77 \\
\end{array}$ & $\begin{array}{l}2,23 \\
2,33 \\
2,11 \\
2,10 \\
1,89 \\
2,07 \\
2,39 \\
2,03 \\
1,00 \\
2,04\end{array}$ \\
\hline math & & 1.13 & 3,61 & 5,09 & 1.25 & 1.55 & 1,88 & 1,09 & 3,4 & 6.87 & 1.17 & $1, \infty$ & $1,8 \mathrm{~A}$ & 1.22 & 1.81 & 2.02 \\
\hline Valor de $\mathrm{F}$ & & $\ddot{*}$ & $\ddot{*}$ & $\ddot{*}$ & $\ddot{*}$ & $*$ & $*$ & ns & 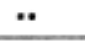 & $\because$ & $\cdots$ & $*$ & $* \cdot$ & *. & ns & as \\
\hline DMS. Tutery SW & & $\begin{array}{l}0.66 \\
0.78 \\
\end{array}$ & $\begin{array}{l}2,68 \\
3,18 \\
\end{array}$ & $\begin{array}{l}3,06 \\
\mathbf{3 , 6 3} \\
\end{array}$ & $\begin{array}{l}0,79 \\
0,94 \\
\end{array}$ & $\begin{array}{l}1,04 \\
1,23 \\
\end{array}$ & $\begin{array}{l}1,56 \\
1,85 \\
\end{array}$ & $\overline{-}$ & $\begin{array}{l}3.59 \\
4,26 \\
\end{array}$ & $\begin{array}{r}3.99 \\
4.73 \\
\end{array}$ & $\begin{array}{l}0,50 \\
0.59 \\
\end{array}$ & $\begin{array}{l}1.11 \\
1.31 \\
\end{array}$ & $\begin{array}{l}1,33 \\
1,58 \\
\end{array}$ & $\overline{-}$ & $\overline{-}$ & $\overline{-}$ \\
\hline C.V. (s) & & 27,31 & $3, n$ & 28,11 & 29,56 & 31,23 & 39.72 & 32.50 & 30,22 & 27,18 & 20,06 & 30,78 & 33,95 & 22.35 & 30,48 & 31,81 \\
\hline
\end{tabular}

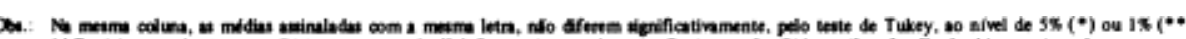

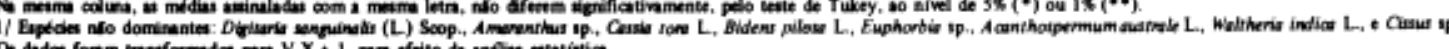


QUADRO 8 - Resultados médios do número total de plantas daninhas por espécies numa área de $5,00 \mathbf{m}^{2}$, na cultura algodoeira (G. hirsutum L.), cultivar «Minas Dona Beja» em Sete Lagoas, Zona Metalúrgica, Minas Gerais, no ano agricola de 1974/75.

\begin{tabular}{|c|c|c|c|c|c|c|c|c|c|c|c|c|c|c|c|c|c|c|c|}
\hline \multirow{2}{*}{ Intamets: } & \multirow{2}{*}{ 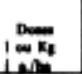 } & \multicolumn{3}{|c|}{ comentax } & \multicolumn{3}{|c|}{ Amstar abous } & \multicolumn{3}{|c|}{ 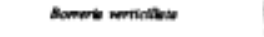 } & \multicolumn{3}{|c|}{ tromesen in. } & \multicolumn{3}{|c|}{ 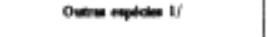 } & \multicolumn{3}{|c|}{ 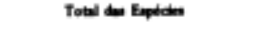 } \\
\hline & & 14 & 20 & 34 & is & 24 & is & 14 & 20 & 3. & 1. & 2 & 3. & 1. & ${ }^{24}$ & 34 & 14 & 20 & 34 \\
\hline 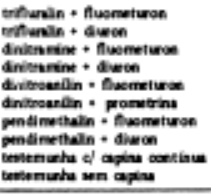 & 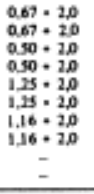 & $\begin{array}{l}1,4 \\
1,92 \\
1,77 \\
1,35 \\
1,0 \\
1,74 \\
1,7 \\
1,70 \\
1,00 \\
1,90 \\
\end{array}$ & 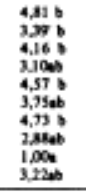 & 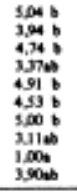 & 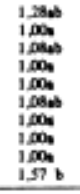 & 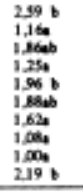 & 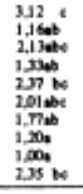 & $\begin{array}{c}1000 \\
1000 \\
1000 \\
1000 \\
1000 \\
1000 \\
1000 \\
1000 \\
1,000 \\
1000 \\
1000 \\
1000\end{array}$ & 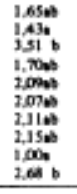 & 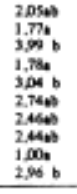 & 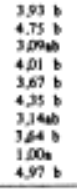 & 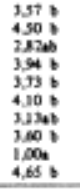 & 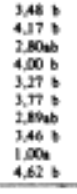 & 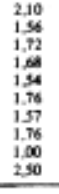 & 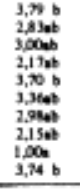 & 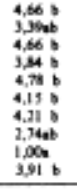 & 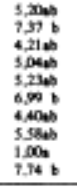 & 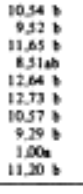 & 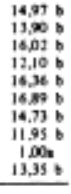 \\
\hline ma. & & 1.62 & 2.61 & 306 & 1,16 & 1,66 & 1,94 & 1.10 & 2,04 & 2.42 & 3.6s & 3.91 & 3,39 & $n$ & $2 n$ & & s.s. & $2, \mathrm{~m}$ & 13,13 \\
\hline Vwax \& F F & & $\Delta$ & .* & $*$ & $\cdot$ & $*$ & $\cdot \cdot$ & ** & $*$ & $*$ & $*$ & .* & $\cdot \cdot$ & nst & $\cdot \cdot$ & & $*$ & $* *$ & $*$ \\
\hline 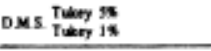 & & - & $\begin{array}{l}2.38 \\
2,92\end{array}$ & $\begin{array}{l}2.69 \\
2,93 \\
\end{array}$ & $\begin{array}{l}0.53 \\
0.03 \\
\end{array}$ & $\begin{array}{l}0.0,0 \\
0.9 \\
\end{array}$ & $\begin{array}{l}1.92 \\
1.21 \\
\end{array}$ & $\begin{array}{l}0,3,3 \\
0,44 \\
\end{array}$ & $\begin{array}{l}1,65 \\
1,95 \\
\end{array}$ & 1,4 & 235 & $\begin{array}{l}19 a \\
235 \\
\end{array}$ & $\begin{array}{l}1.87 \\
2.27 \\
\end{array}$ & $=$ & $\begin{array}{l}2,03 \\
2,41 \\
\end{array}$ & & $\begin{array}{l}396 \\
4.72 \\
\end{array}$ & in & $\begin{array}{l}7.16 \\
0.40 \\
\end{array}$ \\
\hline Cr. (s) & & $32 \mathrm{pt}$ & $\mathbf{x}, n$ & 8,21 & 22,67 & 22,35 & $25, .22$ & 15,76 & 37,80 & $31,6 \mathrm{~s}$ & 28.79 & 26,46 & 26.14 & 4.97 & 3304 & & 38.66 & 32.04 & 2530 \\
\hline
\end{tabular}

QUADRO 9 - Resultados médios obtidos nas análises tecnológicas da fibra do algodão no ensaio de competição de misturas de herbicidas, no ano agricola de 1974/75.

\begin{tabular}{|c|c|c|c|c|c|c|c|c|c|c|c|c|c|c|c|c|}
\hline \multirow{2}{*}{ Trotumantex } & \multirow{2}{*}{ 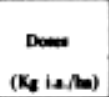 } & \multicolumn{3}{|c|}{ 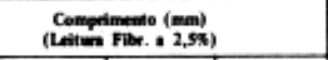 } & \multicolumn{3}{|c|}{$\begin{array}{l}\text { Uwifoemendede de } \\
\text { Comprimento }\end{array}$} & \multicolumn{3}{|c|}{ 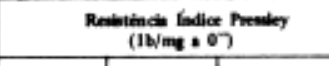 } & \multicolumn{3}{|c|}{$\begin{array}{l}\text { Finas } \\
\text { Intlice Haronaim }\end{array}$} & \multicolumn{3}{|c|}{$\begin{array}{c}\text { Maturdade } \\
\text { (Mikrouctoplo) }\end{array}$} \\
\hline & & Cophopolt & Pontentinth & Stent Lapen & Capmosto & Morterines. & Sete Layou & Caplenopolte & Portetinth & Sete laspos & Coplespolth & Ponterituns & Sete Lasou & Copletopolt & Pontedriber & Stet Lenow \\
\hline 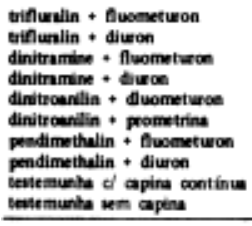 & 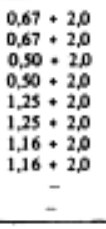 & $\begin{array}{l}26.9 \\
26,7 \\
22,9 \\
26,2 \\
25,7 \\
25,9 \\
26.2 \\
26.8 \\
25,9 \\
25,8 \\
\end{array}$ & 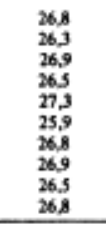 & 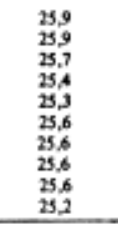 & $\begin{array}{l}48,4 \\
49,1 \\
47,4 \\
47,1 \\
48,3 \\
47,1 \\
48,4 \\
48,4 \\
49,2 \\
47,8\end{array}$ & $\begin{array}{l}49,1 \\
49,2 \\
52,4 \\
50,0 \\
52,7 \\
50,6 \\
49,0 \\
50,1 \\
48,5 \\
45,7 \\
\end{array}$ & $\begin{array}{l}50,3 \\
50,7 \\
51.5 \\
41.4 \\
31.6 \\
49 ., \\
51.2 \\
31.2 \\
49,0 \\
90,3\end{array}$ & $\begin{array}{l}7,8 \\
7,8 \\
79 \\
8,0 \\
7,8 \\
7,9 \\
7,7 \\
79 \\
7,8 \\
79\end{array}$ & $\begin{array}{l}7,8 \\
7,7 \\
7,6 \\
7,7 \\
7,4 \\
7,5 \\
7,5 \\
7,4 \\
7,5 \\
7,4\end{array}$ & $\begin{array}{l}7,0 \\
7,9 \\
7,9 \\
7,4 \\
7,7 \\
7,6 \\
7,6 \\
7,5 \\
7,3\end{array}$ & $\begin{array}{l}3,9 \\
4,2 \\
4,3 \\
4,1 \\
3,9 \\
3,9 \\
4,0 \\
5,0 \\
4,0 \\
3,7\end{array}$ & $\begin{array}{l}3.8 \\
4.0 \\
39 \\
39 \\
3,8 \\
3,9 \\
39 \\
4,4 \\
39 \\
38\end{array}$ & $\begin{array}{l}3.7 \\
40 \\
3.8 \\
3,8 \\
3.8 \\
3,8 \\
3.8 \\
3.8 \\
3.8 \\
3.8\end{array}$ & $\begin{array}{l}82,2 \\
82,0 \\
8550 \\
85,6 \\
82.6 \\
85,6 \\
84,2 \\
85.6 \\
82,2 \\
81,2 \\
\end{array}$ & $\begin{array}{l}83 \\
34 \\
80 \\
50 \\
79 \\
78 \\
50 \\
83 \\
46 \\
81\end{array}$ & $\begin{array}{l}84.2 \\
86,2 \\
81.2 \\
86.4 \\
82.8 \\
82.4 \\
86,8 \\
80.0 \\
81.4 \\
80,2\end{array}$ \\
\hline Media & & 26,3 & 26,7 & 25,6 & 48,1 & 49.7 & 90,6 & 7,8 & 7,6 & 7,5 & 40 & 39 & 38 & 83,6 & 81 & 83,2 \\
\hline Valor \&e "F" & & ns & ns & as & ns & ns & s & ns & ns & ns & ns & as & as & $n a$ & ns & ns \\
\hline cV. (n) & & 4.04 & 3.67 & 2.68 & 6.33 & 6.54 & 5,47 & 395 & 4,00 & 8,61 & 701 & 12,04 & 2,64 & 6.11 & 4,74 & 5.93 \\
\hline
\end{tabular}


QUADRO 10 - Resultados médios do ensaio de competição de misturas de herbicidas na cultura algodoeira, (G. hirsutum L.), utilizando-se a es cala European Weed Research Council, (E.W.R.C.), no ano agrícola de 1974/75.

\begin{tabular}{|c|c|c|c|c|c|c|c|c|c|c|}
\hline \multirow{3}{*}{ Tntamentos } & \multirow{3}{*}{$\begin{array}{c}\text { Doses } \\
\text { (Kg i.a./ha) }\end{array}$} & \multicolumn{9}{|c|}{ Médias da E.W.R.C. (1-9) } \\
\hline & & \multicolumn{3}{|c|}{ Primeira avaliaçäo 1/ } & \multicolumn{3}{|c|}{ Segunda avaliação $2 /$} & \multicolumn{3}{|c|}{ Tercein avaliaçio $3 /$} \\
\hline & & Capinópolis & Porteirinka & Sete Lagoas & Capinópolis & Porteirinha & Sete Lagoas & Capinópolis & Porteirinha & Sete Lagoas \\
\hline $\begin{array}{l}\text { trifluralin + fluometuron } \\
\text { trifluralin + diuron } \\
\text { dinitramine + fluometuron } \\
\text { dinitramine + diuron } \\
\text { dinitroanilin + fluometuron } \\
\text { dinitroanilin + prometrina } \\
\text { pendimethalin + fluometuron } \\
\text { pendimethalin + diuron } \\
\text { testemunha c/ capina contínua } \\
\text { testemunha sem capina }\end{array}$ & $\begin{array}{c}0,67+2,0 \\
0,76+2,0 \\
0,50+2,0 \\
0,50+2,0 \\
1,25+2,0 \\
1,25+2,0 \\
1,16+2,0 \\
1,16+2,0 \\
- \\
-\end{array}$ & $\begin{array}{l}6,2 \mathrm{~b} \\
7,6 \mathrm{ab} \\
6,8 \mathrm{ab} \\
7,0 \mathrm{ab} \\
7,6 \mathrm{ab} \\
6,6 \mathrm{~b} \\
6,0 \mathrm{~b} \\
7,0 \mathrm{ab} \\
9,0 \mathrm{a} \\
1,0 \mathrm{c}\end{array}$ & $\begin{array}{l}7,2 \mathrm{~b} \\
7,6 \mathrm{ab} \\
8,0 \mathrm{a} \\
7,2 \mathrm{~b} \\
7,6 \mathrm{ab} \\
6,8 \mathrm{~b} \\
7,8 \mathrm{ab} \\
7,0 \mathrm{~b} \\
9,0 \mathrm{a} \\
3,6 \mathrm{c}\end{array}$ & $\begin{array}{l}6,8 \mathrm{ab} \\
5,8 \mathrm{~b} \\
7,4 \mathrm{ab} \\
6,0, \mathrm{~b} \\
6,2 \mathrm{~b} \\
5,4 \mathrm{~b} \\
6,8 \mathrm{ab} \\
6,2 \mathrm{~b} \\
9,0 \mathrm{a} \\
1,0 \mathrm{c}\end{array}$ & $\begin{array}{l}5,6 \mathrm{~b} \\
7,0 \mathrm{ab} \\
6,6 \mathrm{ab} \\
6,2 \mathrm{ab} \\
8,2 \mathrm{a} \\
6,4 \mathrm{ab} \\
5,6 \mathrm{~b} \\
6,4 \mathrm{ab} \\
9,0 \mathrm{a} \\
1,0 \quad \mathrm{c}\end{array}$ & $\begin{array}{l}5,4 \mathrm{~b} \\
5,6 \mathrm{~b} \\
6,0 \mathrm{ab} \\
5,0 \mathrm{~b} \\
6,2 \mathrm{ab} \\
4,4 \mathrm{bc} \\
6,2 \mathrm{ab} \\
5,6 \mathrm{~b} \\
9,0 \mathrm{a} \\
1,2 \mathrm{c}\end{array}$ & $\begin{array}{l}5,2 \mathrm{~b} \\
4,8 \mathrm{~b} \\
6,0 \mathrm{ab} \\
5,6 \mathrm{~b} \\
5,2 \mathrm{~b} \\
5,4 \mathrm{~b} \\
6,2 \mathrm{ab} \\
6,0 \mathrm{ab} \\
8,6 \mathrm{a} \\
1,0 \mathrm{c}\end{array}$ & $\begin{array}{l}5,2 \mathrm{ab} \\
5,4 \mathrm{ab} \\
5,0 \mathrm{~b} \\
4,8 \mathrm{bc} \\
6,4 \mathrm{ab} \\
5,4 \mathrm{ab} \\
4,4 \mathrm{bc} \\
5,2 \mathrm{ab} \\
9,0 \mathrm{a} \\
1,0 \mathrm{c}\end{array}$ & $\begin{array}{l}4,8 \mathrm{~b} \\
4,8 \mathrm{~b} \\
5,4 \mathrm{ab} \\
5,0 \mathrm{~b} \\
5,2 \mathrm{~b} \\
4,0 \mathrm{bc} \\
5,0 \mathrm{~b} \\
5,2 \mathrm{~b} \\
9,0 \mathrm{a} \\
1,0 \mathrm{c}\end{array}$ & $\begin{array}{l}4,0 \mathrm{~b} \\
4,0 \mathrm{~b} \\
3,8 \mathrm{bc} \\
5,0 \mathrm{~b} \\
4,4 \mathrm{~b} \\
3,8 \mathrm{bc} \\
4,2 \mathrm{~b} \\
4,8 \mathrm{~b} \\
9,0 \mathrm{a} \\
1,8 \mathrm{c}\end{array}$ \\
\hline Média & & 6,5 & 7,2 & 6,1 & 6,2 & 5,5 & 5,4 & 5,2 & 4,9 & 4,5 \\
\hline Valor de F & & $\bullet$ & $\bullet$ & $\bullet$ & $* *$ & $4 *$ & $* *$ & $\cdots$ & $*$ & $* *$ \\
\hline D.M.S. & & 2,5 & 1,9 & 2,7 & 2,9 & 3,5 & 2,8 & 3,8 & 3,6 & 2,1 \\
\hline C.V. (\%) & & 15,46 & 10,46 & 17,50 & 18,35 & 25,33 & 20,52 & 29,05 & 28,80 & 19,01 \\
\hline
\end{tabular}


8. Análise tecnológica da fibra - Os dados obtidos podem ser observados no quadro 9. Pela análise de variância, verificou-se que não houve nenhuma diferença significativa para os dados obtidos nas três regiões estudadas.

\section{AGRADECIMENTOS}

Os autores agradecem aos Srs. Luis Marques e Seymando S. Sarmento por haver cedido a sua propriedade para instalar os ensaios nas Regiões do Triângulo e Norte de Minas, respectivamente e aos Técnicos Agrícolas Srs. Juarez Guimarães, Ernesto Muniz e Edward Antonio Dutra, pela valiosa colaboração na condução deste trabalho.

\section{LITERATURA CITADA}

1. Azevedo, J.J.; Chaves, R. \& Yepes, J.H. Mezcla de herbicidas pré-emergentes para el algodonero (Gossypium hirsutum L.) en el Valle del Cauca. In: Seminário de la Sociedad Colombiana de Control de Malezas y Fisiologia Vegetal (COMALFI), $3 .^{\circ}$, Palmira, Colombia, 1971. Resumenes, p. 10-11.

2. Alves, A. \& Forster, R. Nitralin na cultura do algodoeiro. Efeitos de doses e da profundidade de incorporaçáo ao solo. Divulgaçáo Agronômica, 25: 18-11, 1968.

3. Alves, A.; Forster, R. \& Gregori, R. Variaçōes nos métodos de aplicaçâo dos herbicidas diuron e trifluralin na cultura do algodoeiro. In: Seminário Brasileiro de Herbicidas e Ervas Daninhas, 6..$^{\circ}$, Sete Lagoas, 1966. Resumos, p. 51-64.

4. Azzi, G.M. \& Fernandes, J. Método de julgamento do efeito herbicida. In: Seminário Brasileiro de Herbicidas e Ervas Daninhas, 6. ${ }^{\circ}$, Sete Lagoas, 1966. Resumos, p. 21-29.

5. Begazo, J.C.O. \& Sediyama, T. Comparaçâo de herbicidas e métodos de aplicaçáo na cultura do algodoeiro (Gossypium hirsutum L.) Revista Ceres. U.F.V., Viçosa, M.G. 17 (8): 294-302, 1971.

6. Chaves, E. Evaluación del herbicida CP-50144 en tratamento post-emergente sobre el cultivo del algodonero. In: Seminário de la Sociedad Colombiana de Control de Malezas y Fisiologia Vegetal (COMALFI), 1.․, Bogotá, Colömbia, 1969. Resumenes, p. 10-11.
7. Corrales, M.A. Control químico de malezas en algodonero (Gossypium hirsutum L.), Investigaciones Agropecuárias del Peru. Dirección General de Investigaciones Agropecuárias. Lima, Perú. 1 (2): 14-20, 1970.

8. Cordova, S.G. Control químico de malezas en el cultivo del algodonero (Gossypium hirsutum L.), cultivar *Del Cerro*. In: Convención Nacional de Controladores de Malezas, $10^{\circ}$, Chiclayo, Perú. 1972. Resumenes, p. 35-37.

9. Cruz, L.S.P. \& Leiderman, L. Efeito comparativo de dois novos herbicidas no controle de ervas daninhas na cultura de algodão. In: Seminário Brasileiro de Herbicidas e Ervas Daninhas, 9., Campinas, SP. 1972. Resumos, p. 36.

10. Cruz, L.S.P. \& Leiderman, L. Primeiros estudos comparativos de efeito herbicida de fluochloralin e dinitramine para algodoeiro. O Biólogo. Sáo Paulo, SP. 40 (11): 314-320, 1974.

11. Cruz, L.S.P. \& Leiderman, L. Dinitramine: novo herbicida de pré-plantio incorporado para algodoeiro. In: Reuniảo Anual da S.B.P.C., 27. ${ }^{\circ}$ Seção L. Agricultura. Belo Horizonte, MG. 1975. Resumos, $53 \mathrm{~L}$.

12. Estrada, R.J. Estudio económico y de factibilidade del uso de herbicida en cultivo del algodonero (Gossypium hirsutum L.), variedad -Del Cerro*, em el Departamento de Lambaveque, Perú (Tese de grado). (mimeografado), 1972. 63p.

13. Laca-Buendia J.P. del C.; Penna, V.J.C. \& Ferreira, B.M. Competição de herbicidas na cultura algodoeira (Gossypium hirsutum L.), no Triângulo Mineiro. In: Seminário Brasileiro de Herbicidas e Ervas Daninhas, 10. ${ }^{\circ}$ Santa Maria, RS. 1974. Resumos, p. 15-16.

14. Leiderman, L.; Lobato, C.A. \& Silveira, R.I. Aplicaçâo de herbicidas em algodáo em três Regiōes do Estado de Sảo Paulo. O Biólogo. São Paulo, SP. 31 (8): 168-175, 1965.

15. Leiderman, L.\& Santos, C.A.L; Figueiredo, P.E. \& Silveira, R.I. Controle de ervas daninhas do algodão com misturas de trifluralin e diuron em quatro regióes de Sáo Paulo. O Biólogo. Sáo Paulo, SP. 32 (7): 158-162, 1966.

16. Ometto, D.A. \& Abreu, de C.P. Aplicação de herbicidas na cultura do algodão. In: Seminário Brasileiro de Herbicidas e Ervas Daninhas e I Reuniáo Latinoamericana de Luta Contra as Ervas Más, 4. ${ }^{\circ}$, Rio de Janeiro, RJ. 1962. Resumos, p. 209-217.

17. Saldarriaga, A.; Cruz de La, R. \& Lagos, E. Estudio de herbicidas en algodón. In: Seminário de la Sociedade Colombiana de Control de Malezas y Fisiologia Vegetal (COMAL - 
FI), $1 .^{\circ}$, Bogotá, Colombia. 1969. Resumenes, p. 17-19.

18. Santos, C.A.L.; Leiderman, L. \& Figueiredo, P. Ensaios comparativos entre cotoran, novo herbicida à base de uréia, diuron e trifluralin na cultura do algodāo. In: Seminário Brasileiro de Herbicidas e Ervas Daninhas, 6. Sete Lagoas, MG, 1966. Resumos, p. 65-71.

19. Santos, C.A.L. Aplicaçāo de herbicidas em pré-plantio e pré-emergência na cultura do algodáo. In: Seminário Brasileiro de Herbicidas e Ervas Daninhas, 8. ${ }^{\circ}$, Botucatu, SP, 1970. Resumos, p. IX-02.

20. Santos, C.A.L. \& Hertwig, K.V. Emprego de herbicidas em pós emergência na cultura do algodảo. In: Seminário Brasileiro de Herbicidas e Ervas Daninhas, 8. ${ }^{\circ}$, Botucatu, SP. 1970. Resumos, p. IX-03.

21. Savage, K.E. \& Bardsley, E. Relation of time and pré-emergence herbicides to yield of cotton and weeds. Agronomy Journal, 56: 269-271, 1966.

22. Silva, J.B.; Coelho, J.P.; Falliere, J. \& Saturnino, M.A.C. Emprego de herbicidas na cultura do algodảo (Gossypium hirsutum L.). Sete Lagoas, MG. IPEACO. (Série Pesquisa/ Extensão). (mimeografado), 1971. 8p.
23. Torrado, G.E. \& Cardenas, J. Mezclas de herbicidas y nuevos productos para el control de malezas en algodón. In: Seminàrio de la Sociedade Colombiana de Control de Malezas y Fisiologia Vegetal (COMALFI), 3.", Palmira, Colombia, 1971. Resumenes, p. 10-11.

24. Torrado, G.E. \& Cardenas, L. Control postemergente de malezas en algodón. In: Seminário de la Sociedad Colombiana de Control de Malezas y Fisiologia Vegetal (COMALFI), 3.․, Palmira, Colombia, 1971. Resumenes, p. 15-17,

25. Yepes, J.H. \& Chaves, R. Herbicidas pré-emergentes en tratamento de presiembra incorporado para el algodonero. In: Seminário de la Sociedad Colombiana de Control de Malezas y Fisiologia Vegetal (COMALFI), 2.' Bogotá, Colombia, 1970. Resumenes, p. $48-49$.

26. Yepes, J.H. Herbicidas post-emergentes dirigidos en el algodonero: In: Seminário de la Sociedad Colombiana de Control de Malezas y Fisiologia Vegetal (COMALFI), 3. Palmira, Colombia, 1971. Resumenes, p. 9-10. 